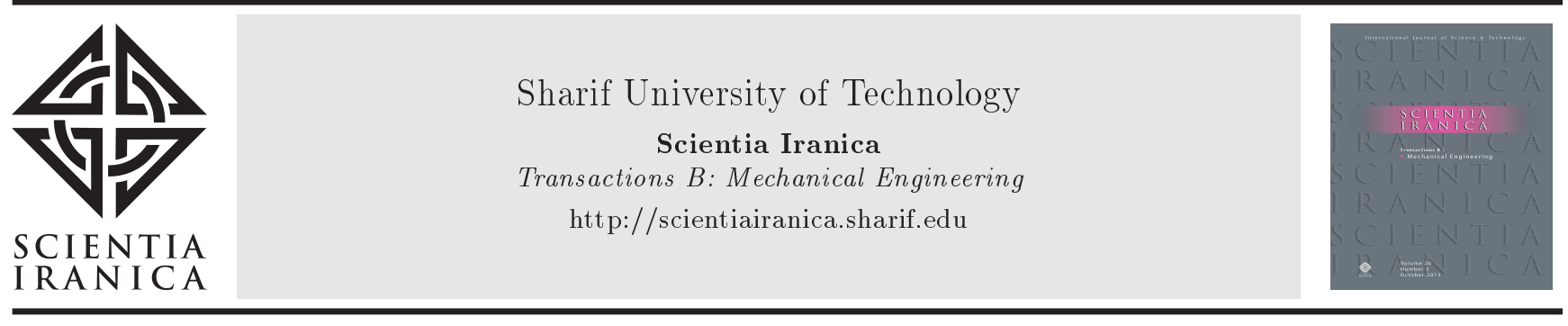

\title{
TF/TA optimal flight trajectory planning using a novel regenerative flattener mapping method
}

\author{
A. Kosari* and S.I. Kassaei \\ Aerospace Group, Faculty of New Sciences and Technologies, University of Tehran, Tehran, Iran.
}

Received 8 July 2018; received in revised form 9 November 2018; accepted 5 January 2019

\begin{abstract}
KEYWORDS
Trajectory planning;

Terrain following;

Terrain avoidance;

Low altitude flight;

Schwarz-Christoffel

mapping;

Virtual terrain model;

Optimal control;

Direct pseudospectral

method.
\end{abstract}

\begin{abstract}
In this paper, a new methodology is proposed to enhance the conformal mapping applications in the process of optimum trajectory planning in Terrain Following (TF) and Terrain Avoidance (TA) Flights. The new approach uses the conformal mapping concept as a flattener tool to transform the constrained trajectory-planning problem with flight altitude restrictions due to the presence of obstacles into a regenerated problem with no obstacle and minimal height constraints. In this regard, the Schwarz-Christoffel theorem was utilized to incorporate the height constraints into the aircraft dynamic equations of motion. The regenerated optimal control problem was then solved by a numerical method, namely the direct Legendre-Gauss-Radau pseudospectral algorithm. A composite performance index of flight time, terrain masking, and aerodynamic control effort was optimized. Furthermore, to obtain realistic trajectories, the aircraft maximum climb and descent rates were imposed as inequality constraints in the solution algorithm. Several case studies for two-dimensional flight scenarios show the applicability of this approach in TF/TA trajectory planning. Extensive simulations confirm the efficiency of the proposed approach and verify the feasibility of solutions, satisfying all of the constraints underlying the problem.

(C) 2020 Sharif University of Technology. All rights reserved.
\end{abstract}

\section{Introduction}

According to the safety reports published by International Civil Aviation Organization (ICAO)[1], a great percentage of flight accidents over the last two decades have occurred during the approach and landing phases of flight or due to the Loss of Control (LOC) at lowaltitude flights. LOC and Controlled Flight into Terrain (CFIT) are the major contributors to fatal aircraft accidents around the world. Safety issues are even more acute when the aircraft is flying at a low altitude. In terminal phases of flight in which the aircraft decreases

*. Corresponding author. Tel./Fax: +982161118495 E-mail addresses: kosari_a@ut.ac.ir (A. Kosari); imankassaei@ut.ac.ir (S.I. Kassaei)

doi: $10.24200 /$ sci.2019.51314.2109 its altitude or during aborted landing where the pilot conducts a so-called "go-around" maneuver, especially in mountainous regions or close to metropolitan areas, the aircraft might be expected to perform a safe Terrain Following (TF) or Terrain Avoidance (TA) maneuver.

Accordingly, aircrafts with TF/TA capabilities are able to avoid sudden crashes caused by the collision with terrains while flying at low altitudes near the ground surface. The Terrain Avoidance Warning Systems (TAWS) currently used in some civil airplanes provide the pilot with a warning of impending obstacles or probable CFIT. TAWSs usually utilize a combination of sensors data and digital map database as their inputs.

In addition to manned airplanes, Unmanned Aerial Vehicles (UAVs) have been extensively used to carry out various missions in recent years. UAVs offer major advantages in missions in dull, dirty, or 
dangerous places, such as the inspection of terrain, pipelines, utilities and buildings, search and rescue operations, firefighting, etc. [2]. In such missions, a flying vehicle might be required to fly at low altitudes. The Guidance, Navigation, and Control (GNC) subsystem plays an important role in the success of these missions. Acquiring an autonomous flying vehicle requires transferring the operational responsibilities and functions, including the GNC tasks, from the ground station to the aircraft onboard computer. This causes a UAV to gain greater autonomy and authority, and human agents change from operators into supervisors. As a result, TF/TA trajectory planning requires a sense-and-avoid subsystem incorporated in the GNC subsystem.

As mentioned before, the TAWS in a manned aircraft provides the pilot with a warning of impending CFIT, and some qualitative advisory information about TF flight is shown to the pilot. However, such devices might not provide the necessary warning at the right time and the pilot would not have enough time for an appropriate decision and reaction. Therefore, in order to assist the pilot with taking the appropriate decision and get the UAV to conduct a TF/TA maneuver autonomously, the autopilot might be upgraded by a TF/TA mode by taking the required maneuver and even applying the necessary commands.

Flight path planning could be done in both online and offline modes. In an offline path planning, the entire path in the mission is computed before the start of the motion, whereas the trajectory to the destination is generated incrementally during the motion in the online path planning [3]. As mentioned before, in several missions, a flying vehicle might be forced to fly at low altitudes with previously unknown obstacles in the environment. Thus, it is required that the motion planning task be implemented online during motion in the flying vehicle.

Traditional path-planning methods offer only two main strategies including (1) geometric path planning and (2) dynamic path planning methods. Geometric methods attempt to add dynamic and path constraints to the algorithm by limiting the maximum accepted load-factor or normal acceleration that resulted in limiting the path curvature. In contrast, the dynamic trajectory planning methods attempt to incorporate the path and dynamic constraints numerically in the numerical solution algorithm or by using the Pontryagin Minimum Principle (PMP) [4]. This research proposes an approach by which simple geometrical shapes using interconnected line segments model the terrain profile or the shape of the obstacle. The concept of the Schwarz-Christoffel conformal mapping is then utilized to incorporate the height constraint into the equation of motion.

The problem of autonomous trajectory planning for UAVs is the subject of a great volume of research works so far. The following section presents an attempt to classify and briefly review various methodologies and approaches introduced in the field of TF/TA trajectory planning. In the section called "Problem statement", the problem of two-dimensional TF/TA trajectory planning is formulated as an optimal control problem. The next section titled "Flattener mapping concept" introduces the Schwarz-Christoffel theorem and explains how to use the concept of conformal mappings for solving an optimal TF/TA trajectoryplanning problem. Several case studies and simulation results are presented in "Case-studies and simulation results" section. The conclusion and outline of future works are drawn in the final section.

\section{Trajectory-planning approaches and related works}

Several researchers have investigated different aspects of trajectory planning in TF/TA maneuvers, and various approaches have been proposed to solve the problem. Generally, trajectory-planning methods for flying vehicles could be categorized in the following.

\subsection{Decoupled trajectory planning}

This approach is based on finding a discrete path in configuration space of vehicle using search algorithms like $A^{*}$ algorithm. The obtained discrete path is then used as a basis to generate a feasible trajectory to satisfy the dynamic constraints of the system. Yang and Zhao [5] investigated the trajectory-planning in an environment with priori known obstacles. In this work, the $A^{*}$ search technique was utilized to obtain discretized trajectory solutions, satisfying some performance constraints of the flying vehicle. In another work, Vachtsevanos et al. [6] proposed a missionplanning algorithm for a rotary-wing flying robot in two steps. At the initial step, the $A^{*}$ search engine searched a map mesh for a route from an initial to the final point in order to avoid stationary obstacles. The generated path was optimized and smoothed by a route filter. A trajectory generator then used this route to plan a feasible trajectory.

A rather different approach comprised of two phases was proposed by Karelahti et al. [7]. Firstly, a direct multiple-shooting algorithm was used to compute an optimal trajectory for a 3DoF aircraft model. A $5 \mathrm{DoF}$ simulation was then applied to evaluate the generated trajectories. At the second phase, the optimization parameters were tuned, correspondingly. In a rather common approach known as "Waypoints (WPs) Guidance", an offline discrete path planner was used to generate a set of WPs. Then, this set of WPs was fitted by a spline for which the acceleration commands satisfied the system constraints. Lee et 
al. [8] proposed a 2D trajectory-planning approach for a UAV that flies through mid-course WPs. The path generation incorporates the steady-state flight for straight-line paths and steady turning flight to generate circular arc trajectories. In another research, with no prior knowledge of WPs, Babaei and Mortazavi [9] offered an online 2D trajectory-planning method. In a recent study, Lin et al. proposed a fast obstacle avoidance algorithm based on the geometric approach. Combining the geometric method and selecting the maneuver start time, the authors claimed a $90 \%$ computation time reduction compared to the current waypoint generation methods [10].

\subsubsection{Optimal control methods}

Trajectory-planning problems can be solved through optimal control theory methods. Optimal control methods consider the constraints on the control variables (e.g., the control surface deflections, thrust magnitude, etc.) by applying the PMP. Dynamical constraints on path and states are also addressed by several methods such as slack variables, penalty function, and interior point method [11]. An optimal control approach was discussed by Menon [12] for a TF flight. Terrain masking and flight time minimization were considered in the performance index, and the mathematical model of terrain was incorporated in the equation of motion to generate optimal trajectories. Malaek and Kosari [13] applied the same approach as Menon's to solve a 3D TF/TA trajectory planning problem. They introduced an approach to incorporate the dynamical capabilities of aircrafts via a cost function. The authors also used an inverse dynamic method to solve a $2 \mathrm{D}$ time optimal $\mathrm{TF}$ trajectory planning [14]. In another similar study, the so-called Energy Constant Trajectories (ECT) were introduced for low-altitude flight trajectory planning in TF/TA maneuvers in mountainous areas [15]. Denton and Jones [16] applied "Dynamic Programming" to generate optimal trajectories. The proposed method discretizes the existing terrain profile before conducting the search for optimal trajectories. In another research work, a closed-loop optimal guidance scheme for firstorder control systems was derived for a spin-stabilized flying vehicle [17].

Researchers also have used direct optimal control methods to solve trajectory-planning problems. Kosari et al. [18] introduced a $2 \mathrm{D}$ path planning approach to generate optimal flight trajectories for an aircraft flying in mountainous regions. In this work, the authors used trapezoidal and Hermite-Simpson methods to discretize the state and control variables. In Williams [19], a direct Legendre Pseudo-spectral Method (LPM) was used to obtain 3D optimal online TF/TA trajectories. To do so, firstly, the optimal control problem was transcribed into a Non-Linear Program (NLP) prob- lem and, then, a Sequential Quadratic Programming (SQP) algorithm was solved the NLP. Another work by Kamyar and Taheri [20] applied a direct sequential method to solve a TF/TA trajectory planning. The control inputs were developed for time-, fuel-, and height-optimal scenarios using a 6DoF dynamic model.

\subsubsection{Heuristic optimization methods}

Artificial heuristic methods such as Genetic Algorithm (GA), Particle Swarm Optimization (PSO), etc. have also been used for path-planning problems. Qing [21] used GA, for the first time, to optimize aircraft path in the course of TF/TA flight considering low-altitude flight and penetration requirements in the cost function. In a work by Nikolos et al. [22], a modified GA was utilized to develop offline and online trajectory planners for UAVs in a 3D terrain environment. The flight path was represented by B-spline curves. In the same line of works, Nikolos and Tsourvelouds [23] used GA to optimize path in cooperative flight scenarios of flying vehicles. In Sun et al. [24], PSO was used to design a flying robot flight path in a $3 \mathrm{D}$ environment. Bspline curves were used for smoothness. The flight path planning in constrained and dynamic environments was modeled as a hybrid optimization problem by Karimi and Pourtakdoust [25]. A PSO-based algorithm was used to generate the optimal trajectories to fly over the terrain and random threats. An integrated optimization of the guidance and control system for a dual spin flying vehicle was presented by Nobahari and Arab Kermani [26] using a metaheuristic algorithm, called Tabu continuous ant colony algorithm.

\subsection{Artificial Intelligence (AI) approaches}

Several recent studies have used a combination of path-planning methods and AI algorithms to improve the real-time aspect of trajectory generation. Kosari et al. [27] considered the application of AI in an online trajectory generation. An inverse dynamic method was used to generate offline trajectories. The offline paths were used to train a multi-layer neural network. In Rahim and Malaek [28,29], an optimal trajectory planning plus a fuzzy approach was proposed for TF/TA flight maneuvers. In this method, the relationship between the gradient of terrain slopes and their derivatives, as well as the flight speed and altitude of the aircraft, was used to construct fuzzy rules. In a rather recent study, Bagherian [30] utilized a fuzzylogic system for a UAV 3D TF/TA trajectory planning, assuming that the terrain data and UAV dynamic constraints were available beforehand.

\subsection{Potential field approaches}

Artificial Potential Field (APF) methods have been utilized for obstacle-avoidance trajectory planning in the field of robotics. APF methods are based on the idea of assigning a potential function to the unob- 
structed space and considering the vehicle's motion as a particle that reacts to repulsive forces of the potential field to achieve obstacle avoidance [31]. In Megherbi and Wolovich [32], a complex potential function was derived, which encoded the position, orientation, and shape of the obstacle and how fast and in which direction it moved. By computing the gradient of the potential function, a feedback control law was derived, given the constant curvilinear velocity. Introducing a complex potential function makes it possible to use the conformal mapping to solve the problem for an arbitrarily shaped obstacle using the solution for a circular obstacle case. In another work by Chuang and Ahuja [33], a potential field model was assigned to free space to yield collision avoidance between an object and an obstacle. In a more recent study, Chen et al. [34] used a combination of APF and optimal control theory to address UAV path planning. By introducing an additional control force to the APF and applying the functional optimization method, the trajectory-planning problem was reformed into a constrained optimization problem. In another work, a combination of GA, Dijkstra searching algorithm, and APF approach was utilized to provide an algorithm that solved a global path planning in an environment with multiple threats [35].

Since the overall evaluation of research works conducted so far, the dynamical feasibility of the path and the selection of an efficient numerical algorithm have been the main issues in TF/TA reference trajectory planning methods. Meanwhile, these research studies lack a generalized and systematic approach to addressing such height-constrained flight missions. Some approaches assume that the environment height constraints due to the terrain profiles and obstacles are known prior to the mission operation $[5,12-14,18]$. In some methods, the model of terrain profile has been incorporated in the kinematics equations of motion, requiring the mathematical model of terrains to be exact and differentiable [12-14]. In some of the optimal control approaches, path constraints are incorporated in the "performance index" or the so-called "cost function". In some other trajectory planning approaches, the path constraints are captured in the numerical optimization algorithms [18-20]. Along with the mentioned methods, some approaches, which claim to be implemented in real time, do not guarantee optimality and dynamical feasibility.

The current research attempts to present a solution to some of the aforementioned problems by creating a Virtual Terrain Model (VTM) based on an existing real terrain model. The novel method is used to generate flying routes over naps of the earth with the application of conformal mapping [36]. In the proposed method, the Schwarz-Christoffel mapping is introduced as a tool for facilitating the consideration of height constraints and incorporating it into the equations of motion. In this regard, the shape of the terrain profile, using the conformal mapping exclusivity as a flattener, could have been mapped into a virtually unoccupied space with no formal height restrictions and barriers. The solution strategy proposed by this article conceptually differs from that discussed by Megherbi and Wolovich [32], in which the authors used a complex potential field to solve the path-planning problem for a circular obstacle case and applied the conformal mapping to obtain a solution for other arbitrary obstacle shapes.

Identifying and calculating proper conformal mapping in accordance with the height constraints of the problem might be one of the major challenges of this approach. In this regard, the main efforts have been made to compute a series of basic terrain profiles based on the facilitator mapping function derived by the Schwarz-Christoffel theorem.

\section{Problem statement}

Since almost all of flying vehicles have a plane of symmetry, it is possible to separate the flight dynamics model of the vehicle's motion from distinct longitudinal and lateral-directional equations of motion. Besides, many researchers have attempted to simplify dynamic models in order to reduce the computational time and efforts, provided that the simplification does not affect the feasibility of solutions. With the same reasoning, this research uses the aircraft's longitudinal equations of motion in the trajectory-planning problem.

As outlined in the previous section, the objective is to provide a solution methodology for the aircraft trajectory-planning problem in a TF/TA flight maneuver. In this regard, the following steps are considered to be taken to solve the problem:

Step 1. Modeling the aircraft flight equations (differential equations constraints) discussed in this section;

Step 2. Modeling obstacles and terrain (flight path constraints);

Step 3. Utilizing the flattener mapping concept (discussed in Section 4) to include the constraints into the flight equations;

Step 4. Solving the optimal trajectory-planning problem (discussed in Section 5).

The aircraft's point-mass longitudinal equations of motion assuming a flat non-rotating earth model could be stated as follows [37]:

$$
\begin{aligned}
& \dot{x}=V \cos \gamma, \\
& \dot{y}=V \sin \gamma,
\end{aligned}
$$




$$
\begin{aligned}
& m \dot{V}=T \cos \alpha-D-m g \sin \gamma \\
& m V \dot{\gamma}=T \sin \alpha+L-m g \cos \gamma
\end{aligned}
$$

where parameters $x, y, V$, and $\gamma$ are defined as the state variables and represent downrange, altitude, velocity, and flight-path angle, respectively. Aerodynamic lift and drag forces are notated by $L$ and $D$ and are defined as follows:

$$
\begin{aligned}
& L=\frac{1}{2} \rho V^{2} S_{r e f} C_{L}, \\
& D=\frac{1}{2} \rho V^{2} S_{r e f} C_{D} .
\end{aligned}
$$

In Eq. (5), $S_{r e f}$ is the aircrafts wing area; $C_{L}$ and $C_{D}$ are lift and drag coefficients, respectively, which are the functions of Mach number, altitude, and Angle of Attach (AoA). The aerodynamic lift and drag coefficients are generally modeled as follows in the form of look-up tables:

$$
\begin{aligned}
& C_{L}=C_{L}(y, M, \alpha), \\
& C_{D}=C_{D}(y, M, \alpha) .
\end{aligned}
$$

The thrust force, $T$, could be defined as follows:

$$
T=T_{\max }(M, y) g(\eta),
$$

where $T_{\max }$ is the maximum thrust, and $\eta$ is throttle setting that alters in the range $0 \leq \eta \leq 1$. The function $g(\eta)$ represents the nonlinear behavior of the engine as a function of Mach number and altitude. Aircraft AoA $\alpha$ and throttle setting $\eta$ are considered as control variables. The AoA is actually controlled by elevator control surfaces. The elevator history could be calculated by computing the time history of AoA and its derivative.

The state and control variables considered for the problem and some performance parameters of the flying vehicle might be restricted due to the inherent technological and physical limitations of the aircraft, e.g., the elevator maximum deflection angle and so forth. These limitations specify the allowed ranges for the state and control variables and performance parameters in which they can vary. The control variable constraints could be defined as follows:

$$
\left\{\begin{array}{l}
\alpha_{\min } \leq \alpha \leq \alpha_{\max } \\
0 \leq \eta \leq 1
\end{array}\right.
$$

The maximum Rate of Climb (RoC) and maximum Rate of Descent (RoD) are among the important performance parameters of an aircraft in the TF/TA maneuvers. These parameters are taken into account in the trajectory planning process as an inequality constraint as follows:

$$
-R o D_{\max } \leq \dot{h} \leq R o C_{\max } .
$$

One of the design tools in the trajectory optimization process is the performance index (cost function). In this study, a composite performance index includes two conflicting requirements: flight time and terrain masking. In order to prevent an abrupt change in the AoA and improve the control surface rate saturation problems, it is recommended that the AoA control effort be included in the cost function.

$$
\begin{aligned}
& J=\int_{t_{0}}^{t_{f}}\left[(1-K)+K\left(y-h_{t r}(x)\right)^{2}+\alpha^{2}\right] d t \\
& 0 \leq K \leq 1,
\end{aligned}
$$

where $h_{t r}(x)$ is the terrain profile height plus an allowable clearance above the terrain at the down range $x$ position.

\section{Flattener mapping concept}

The mathematics of complex variables and conformal mapping is not a new subject as it has had widespread applications in the last few decades. A complex function $w=f(z)$, where $z=x+y i$ and $w=$ $u+w i$ are complex numbers, is conformal in a specified domain if and only if $f^{\prime}(z)$ is non-zero over the entire domain $[38,39]$, i.e., $f^{\prime}(z) \neq 0$. These mappings have some unique features, the most important of which could be outlined as follows [37]:

- Conformal mapping preserves the angle locally between two differentiable arcs, as shown in Figure 2;

- An inverse of conformal mapping is computable; suppose that the mapping $f(z)$ is analytic at point $z_{0}$; then, the function $f$ has an inverse $F$ in the neighborhood of $w_{0}=f\left(z_{0}\right)$

\subsection{Schwarz-Christoffel mapping tool}

In this research, these two important features of the conformal maps are used to develop a method for aircraft flight trajectory planning in TF/TA maneuvers. The main idea in this research is to utilize the SchwarzChristoffel conformal map, which is used to transform the boundary of the virtual terrain (barrier) in $w$-plane to the real axis of $z$-plane and the area over the terrain to the upper part of the real axis.

According to the Schwarz-Christoffel theorem, "the real axis of $w$-plane could be mapped to a piecewise linear boundary of a polygon in z-plane with interior angles at successive vertices $A_{1}, \ldots, A_{n}$ as $\alpha_{1} \pi, \ldots, \alpha_{n} \pi$ and the upper half of $w$-plane mapped to the exterior of the polygon (as shown in Figure 1). This transformation is defined by the following equation:

$$
\begin{aligned}
\frac{d z}{d w}= & C_{1}\left(w-a_{1}\right)^{\alpha_{1}-1}\left(w-a_{2}\right)^{\alpha_{2}-1} \\
& \ldots\left(w-a_{n}\right)^{\alpha_{n}-1} .
\end{aligned}
$$




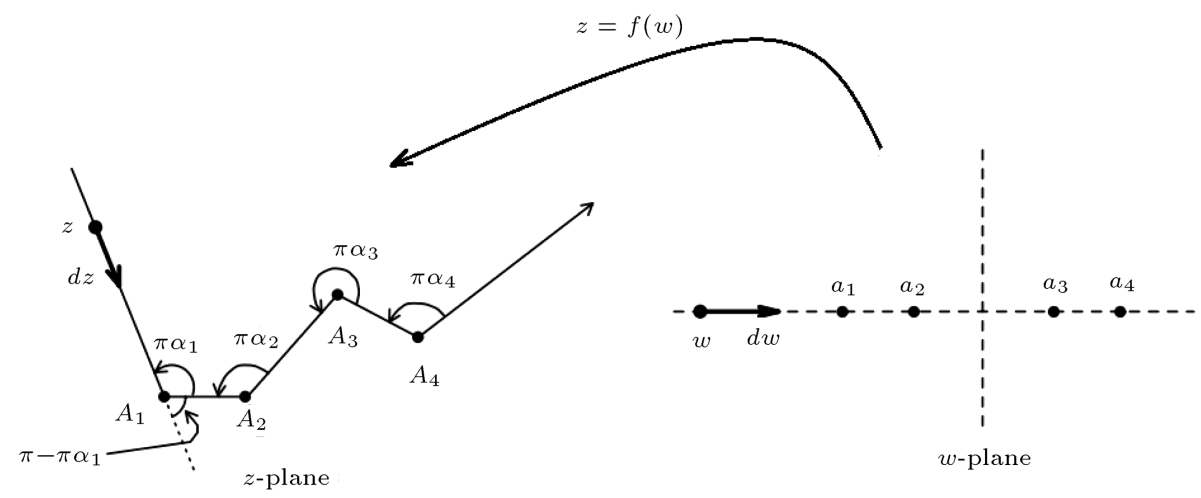

Figure 1. Schwarz-Christoffel theorem.

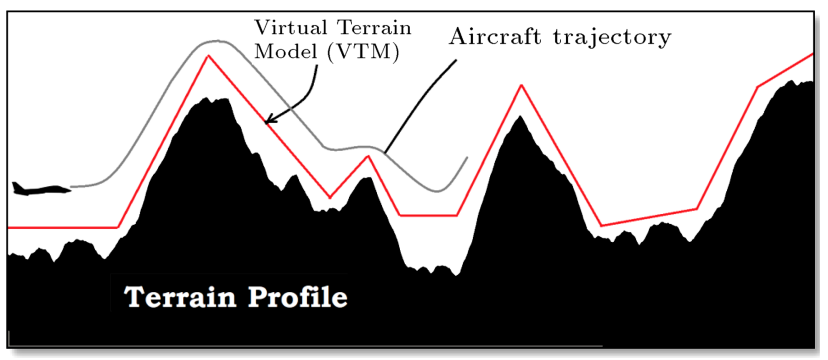

Figure 2. Virtual Terrain Model (VTM) concept based on flattener mapping.

The transformation function, $F$, is computed as follows:

$$
\begin{gathered}
z=F(w)=C_{1} \int_{0}^{w}\left(\left(w-a_{1}\right)^{\alpha_{1}-1} \ldots\left(w-a_{n}\right)^{\alpha_{n}-1}\right) \\
d \zeta+C_{2} .
\end{gathered}
$$

The parameters $C_{1}$ and $C_{2}$ are complex constants that must be computed based on the given points. Except for certain cases and simple geometric shapes, the analytical solution of the integral term in Eq. (12) is not possible and must be numerically calculated.

Based on the very interesting feature of this family of functions, the possibility of transforming geometric shapes to a straight line could make the SchwarzChristoffel mapping an effective candidate to model the peculiar shape of terrains. Figure 2 shows the concept of VTM using a polygonal shape. The actual terrain profile could be estimated globally by a polygon of multiple sides or by multiple local simple geometrical shapes such as triangles, pentagon, etc. Accordingly, this paper has applied this exceptional property to the development of easier mathematical decision spaces in solving the complex TF/TA trajectory-planning problems.

In other words, this study applied this technique to model the real shape of the terrain by the simple polygonal shapes. The Schwarz-Christoffel map theorem then transforms the terrain model into a flat plain in an imaginary space. Consequently, in this unobstructed flattened area, the selection of an optimal flyable route using the traditional techniques of guidance and trajectory design may be more attainable and faster.

\subsection{Formulation of the concept}

At this point, we can describe the formulation of the Flattener mapping concept. The discussion begins with a triangular barrier model, which might be used to estimate and model the terrain profiles. Consider determining a function that maps the exterior of a triangle located in the upper half of the $z$-plane, i.e., the vehicle's real motion space, onto the upper half of the $w$-plane, i.e., the vehicle's motion cyberspace (see Figure 3). The Schwarz-Christoffel theorem yields the desired function as follows:

$$
z=C_{1} \int_{0}^{w} \frac{(\zeta)^{\alpha_{2}}}{\left(\zeta-a_{1}\right)^{\alpha_{1}}\left(\zeta-a_{3}\right)^{\alpha_{3}}} d \zeta+C_{2},
$$

where $a_{1}, a_{2}=0$, and $a_{3}$ are the real-valued numbers associated with complex numbers $A_{1}=-k_{1}, A_{2}=h i$, and $A_{3}=k_{2}$, respectively, and the angles $\alpha_{1} \pi, \alpha_{2} \pi$, and $\alpha_{3} \pi$ are the corresponding angles, as shown in Figure 3 .

Figure 4 shows how the horizontal and vertical lines in $w$-plane are changed under the mapping function determined for a typical triangle with parameters $k_{1}=1200, k_{2}=800$, and $h_{o b s}=1500$.

The constant $C_{1}$ and the integral term in Eq. (13) are not analytically computable, and the mapping function must be calculated with some numerical methods. In the case of an isosceles triangle, i.e., $k_{1}=k_{2}=k$, if $a_{1}$ and $a_{2}$ are selected as -1 and 1 , respectively, the constant $C_{1}$ is derived in terms of the gamma function and the mapping function is expressed as follows:

$$
z=\frac{\left(k-h_{o b s} i\right) \sqrt{\pi}}{\Gamma(\beta+1 / 2) \Gamma(1-\beta)} \int_{0}^{w} \frac{\zeta^{2 \beta}}{\left(1-\zeta^{2}\right)^{\beta}} d \zeta+h_{o b s} i,
$$

where $\pi \beta$ is the base angle of the isosceles triangle, $a$ is a real number selected arbitrarily, and $\Gamma$ is 


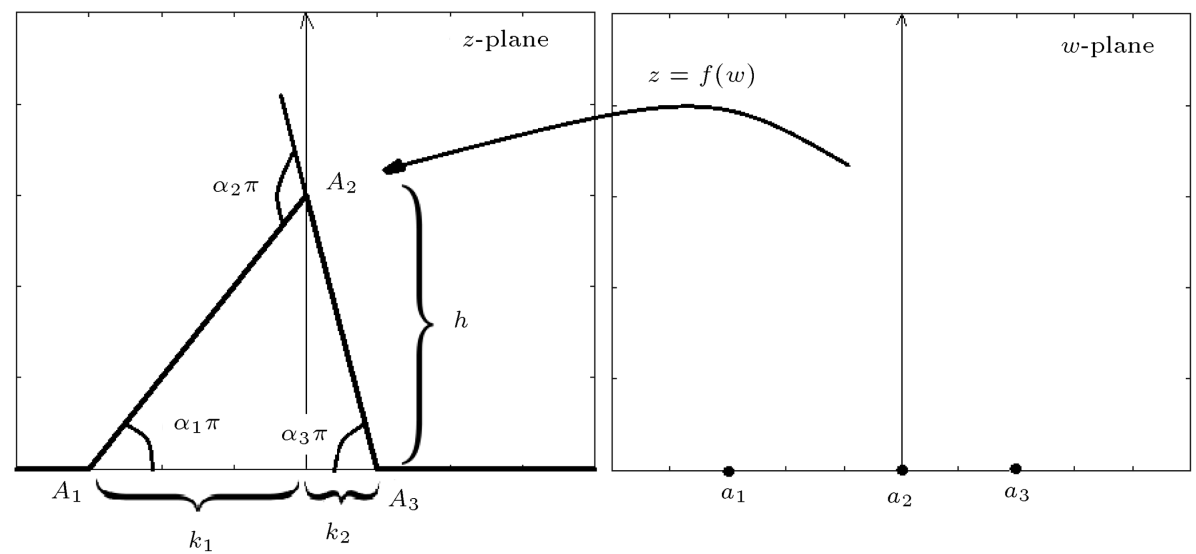

Figure 3. Transformation of the upper half plane into the exterior of a triangular Virtual Terrain Model (VTM).

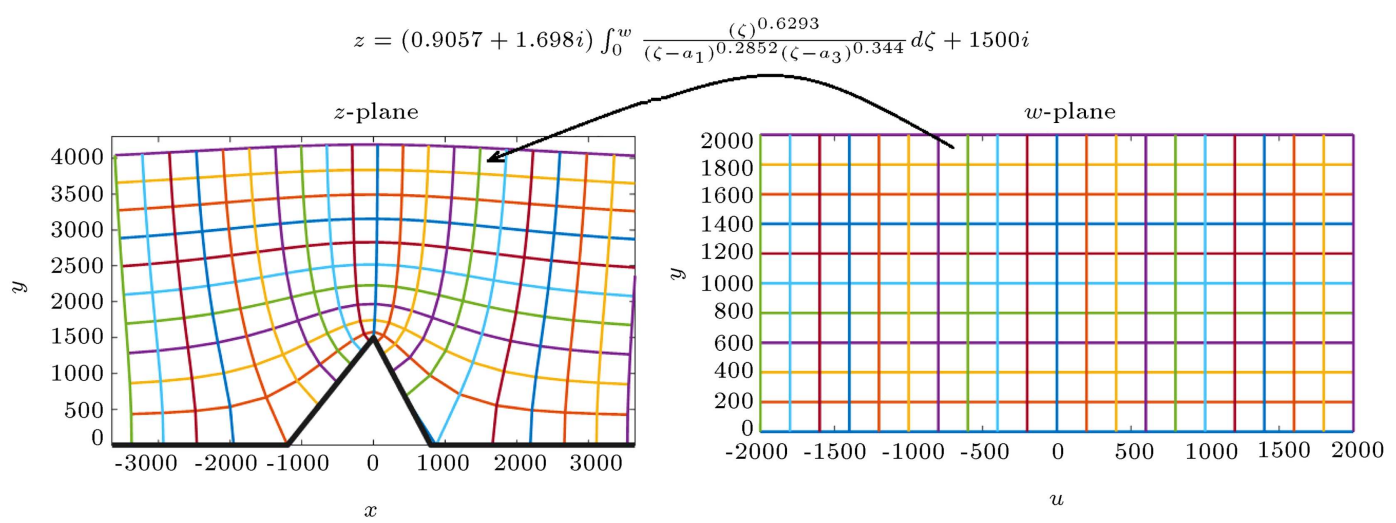

Figure 4. Transformation of the exterior of a triangle $\left(k_{1}=1200, k_{2}=800\right.$, and $\left.h_{o b s}=1500\right)$.

the "gamma function", which is an extension of the factorial function and is defined as follows:

$$
\Gamma(z)=\int_{0}^{\infty} t^{z-1} e^{-t} d t
$$

In the case of the isosceles triangle, if the value of $k$ tends to zero that corresponds to the limit $k \rightarrow 0$ and $\beta \rightarrow \frac{1}{2}$, Eq. (14) is reduced to Eq. (16) [40]:

$$
z=h_{o b s} \sqrt{w^{2}-1^{2}} \text {. }
$$

It was made clear that the transformation function was not always analytically computable, and numerical methods should be used instead. It can also be shown that, in Schwarz-Christoffel transformation, the correspondence of only three points can be prescribed arbitrarily [39]. In other words, $A_{1}, A_{2}$, and $A_{3}$ on the boundaries of the polygon in $z$-plane can be associated with any points $a_{1}, a_{2}$, and $a_{3}$ on the real axis of $w$-plane. If the VTM polygon has more than three vertices, the determination of $a_{4}, a_{5}, \ldots, a_{n}$ might be difficult and must be computed numerically. Various numerical methods are discussed in the literature of mathematics of complex numbers [40,41]. Here, this has developed a Schwarz-Christoffel Terrain Model
Generator (SCTMG) code that generates terrain profiles with greater complexity. The software code uses numerical methods to compute integration constant, the numbers $a_{4}, a_{5}, \ldots, a_{n}$, and the integral term. Figure 5 shows two examples of SCTMG software outputs.

The vehicle's translational equation of motion in $z$-plane could be rewritten as follows:

$$
\dot{z}=\dot{x}+i \dot{y}=V \cos \gamma+i V \sin \gamma
$$

Now, suppose that the mapping function relation of the corresponding VTM is expressed as $z=F(w)$; the equivalent equation of motion in the mapped cyberspace may be expressed as follows:

$$
\begin{aligned}
\dot{w} & =\left(\frac{1}{d z / d w}\right) \dot{z} \\
& =\left(\frac{1}{F^{\prime}(w)}\right)(V \cos \gamma+i V \sin \gamma) .
\end{aligned}
$$

Based on Eq. (18), it is sufficient to determine the implicit derivative of the conformal map, $d z / d w$, in order to implement the transformation process. By taking derivative of Eq. (12) with respect to $w$ and 

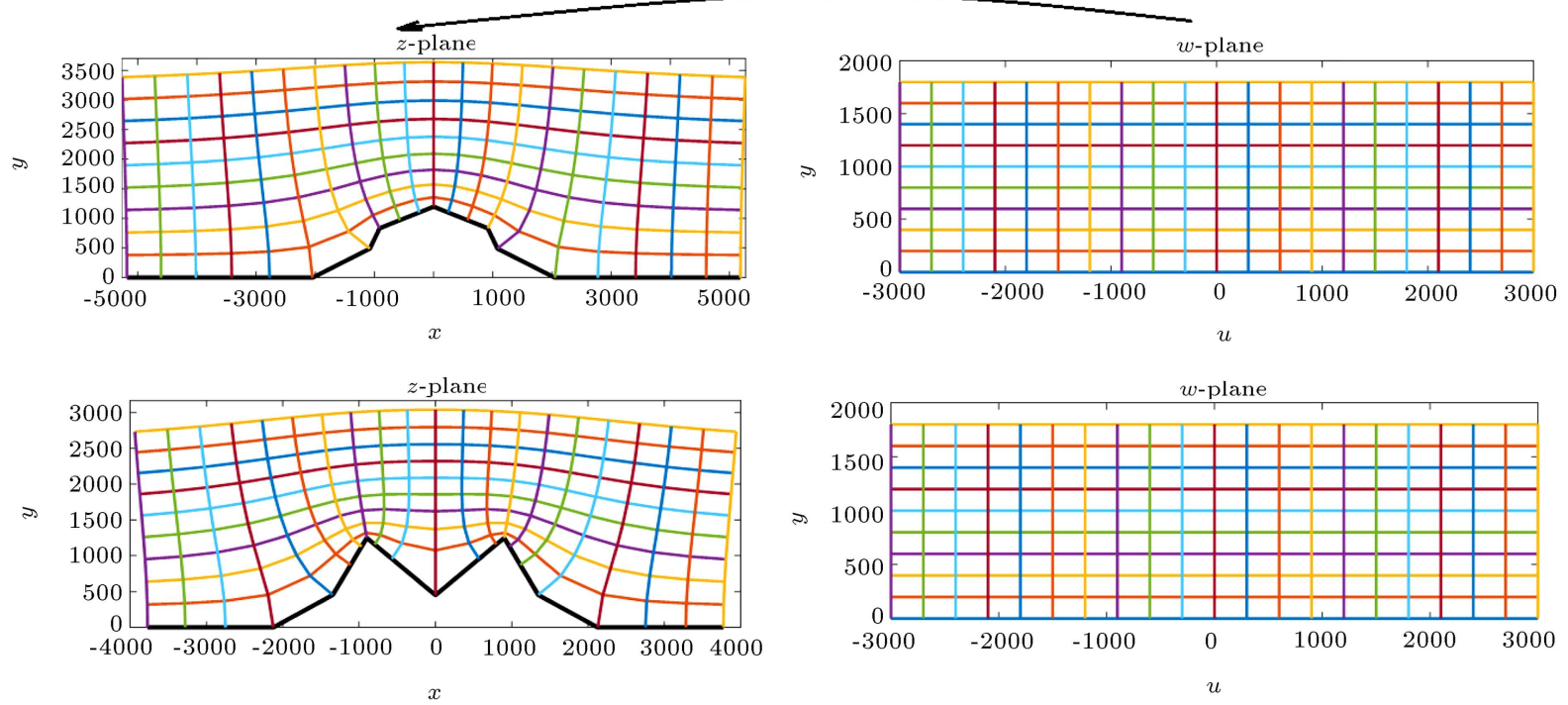

Figure 5. Complex Virtual Terrain Model (VTM) examples.

substituting it in Eq. (18), the flattening equation is be expressed in the form below:

$$
\begin{gathered}
\dot{w}=C_{1}\left(\gamma\left(w-a_{1}\right)^{\alpha_{1}-1} \ldots\left(w-a_{n}\right)^{\alpha_{n}-1}\right) \\
(V \cos \gamma+i V \sin \gamma) .
\end{gathered}
$$

By considering this transformation process, the only constraint on state variables is as follows:

$$
v=\operatorname{imag}(w) \geq H_{\min },
$$

where $H_{\min }$ is a design parameter that can be selected by the trajectory planner. Now, the aircraft flight trajectory planning could be considered as a free final-time optimal control problem expressed as follows: "Based on the flattener-mapping concept explained, Eqs. (19), (3), and (4) form the transformed equations of motion that govern the aircraft's flight trajectory-planning problem. Eqs. (8), (9), and (20) define the problem inequality constraint. The cost function expressed in Relation (10) should be modified in accordance with the transformed problem. Therefore, the cost function that must be minimized in the solution process is expressed as follows":

$$
\begin{aligned}
& J=\int_{t_{0}}^{t_{f}}\left[(1-K)+K\left(v-H_{\min }\right)^{2}+\alpha^{2}\right] d t \\
& 0 \leq K \leq 1 .
\end{aligned}
$$

\section{Solution methodology}

So far, the path-planning problem has been presented as an optimal control problem in the transformed space. Generally, there are two different categories of numerical methods to solve the aforementioned optimal TF/TA trajectory-planning problem, namely indirect and direct [42]. In an indirect method, the calculus of variations in line with the PMP is used to derive the first-order optimality conditions by forming a TwoPoint Boundary Value Problem (TPBVP). Various numerical methods have been developed to solve the TPBVPs [42]. In direct methods, the optimality conditions are not required to be determined prior to the solution. In addition, direct methods have a large radius of convergence and, therefore, do not require an exact initial guess, and there is no need for the guess of costates $[42,43]$. These advantages have recently heightened researchers' interests in the application of direct methods to solve complex optimal control problems.

In this paper, a direct simultaneous approach is used to solve the optimal TF/TA trajectory-planning problem, i.e., both control and state variables are discretized to achieve the NLP. The presented method utilizes the Lagrange polynomials to estimate the state and control variables in the Legendre-Gauss-Radau (LGR) collocation nodes, which are the roots of the sum of the $n$th and $(n-1)$ th degree Legendre polynomials [44-46]. The $n$th degree Legendre polynomial is given as follows:

$$
P_{n}=\frac{1}{2^{n} n !} \frac{d^{n}}{d \tau^{n}}\left[\left(\tau^{2}-1\right)^{n}\right] .
$$

The collocation nodes are defined at the interval of $[-1,1]$. Therefore, the time interval of $\left[t_{0}, t_{f}\right]$ could be mapped to the general interval $\tau \in[-1,1]$ via the linear transformation given by:

$$
\tau=\frac{2 t}{t_{\mathrm{f}}-t_{0}}-\frac{t_{\mathrm{f}}+t_{0}}{t_{\mathrm{f}}-t_{0}} .
$$

The state and control variables are defined as row vectors:

$$
x(\tau) \triangleq\left[\begin{array}{llll}
u(\tau) & v(\tau) & V(\tau) & \gamma(\tau)
\end{array}\right]
$$




$$
u(\tau) \triangleq[\alpha(\tau) \quad \eta(\tau)]
$$

The state is approximated on the basis of Lagrange polynomials given in the following:

$$
L_{k}(\tau)=\prod_{j \neq i}^{N} \frac{\tau-\tau_{j}}{\tau_{i}-\tau_{j}}, \quad i=0, \ldots, N
$$

The state is approximated by a polynomial of degree at most $N$ as:

$$
x(\tau) \approx \mathbf{X}(\tau)=\sum_{k=0}^{N} x\left(\tau_{k}\right) L_{k}(\tau) .
$$

The cost function defined in Eq. (21) could be approximated using the Gauss quadrature approximation as follows:

$$
J \approx \frac{t_{f}-t_{0}}{2} \sum_{k=1}^{N} w_{k}\left[(1-K)+K\left(v-H_{\min }\right)^{2}+\alpha^{2}\right],
$$

where $w_{k}$ represents the corresponding Gauss quadrature weights for the LGR points:

$$
\begin{aligned}
& w_{1}=\frac{2}{N^{2}}, \\
& w_{k}=\frac{1}{\left(1-\tau_{i}\right)\left[\dot{P}_{n-1}\left(\tau_{i}\right)\right]^{2}}, \quad 2 \leq i \leq N .
\end{aligned}
$$

Pseudospectral methods, similar to other direct collocation methods, transform the dynamic differential equations into algebraic equality constraints. This is accomplished by orthogonal collocation, i.e., the derivative of the state approximation in Eq. (26) is collocated with the vector form of differential equations of (Eqs. (3), (9), and (19)). If the converted differential equations of motion are defined by Eq. (29) as shown in Box I, where $R e($.$) and I M($.$) denote the real$ and imaginary parts of a function. The orthogonal collocation could then be written as follows:

$$
\begin{aligned}
& \sum_{k=1}^{N} x\left(\tau_{k}\right) \dot{L}_{k}(\tau)-\frac{t_{f}-t_{0}}{2} f(x(\tau), u(\tau))=0, \\
& k=1, \ldots, N .
\end{aligned}
$$

Eq. (30) can be implemented in the NLP. The finitedimensional NLP associated with the LGR pseudospectral method is then given as follows: minimize Eq.(27)

subjected to Eqs.(8), (9), (20), and (29),

$$
x_{1}=x_{0}, x_{N}=x_{f} .
$$

An optimization algorithm could solve the obtained NLP. In this work, the NLP solver SNOPT [47], which uses the SQP technique, has been used for solving the obtained NLP.

\section{Case studies and simulation results}

Optimal TF/TA trajectory-planning problems are generally defined over a period of time in which the initial time and states (i.e., position, velocity, and flight path angle) of the vehicle are determined; however, the final time and states might be determined or left free based on the flight operational requirements.

The aircraft's mathematical model based on the information provided by Khademi et al. [48] is considered as follows:

$$
\begin{aligned}
C_{L}= & 0.0174+4.3329 \alpha-1.3048 \alpha^{2}+2.2442 \alpha^{3} \\
& -5.8417 \alpha^{4}, \\
C_{D}= & 0.0476-0.1462 \alpha+0.0491 \alpha^{2}+12.8046 \alpha^{3} \\
& -12.6985 \alpha^{4} .
\end{aligned}
$$

The initial and final equality and inequality constraints for a typical TF/TA scenario are considered as follows:

$$
x_{i}=\left\{\begin{array}{l}
x_{i}=\text { specified } \\
y_{i}=\text { specified } \\
V_{i}=\text { specified } \\
\gamma_{i}=\text { specified }
\end{array} \quad x_{f}=\left\{\begin{array}{l}
x_{f}=\text { specified } \\
y_{f}=\text { specified } \\
V_{f}=\text { bounded } \\
\gamma_{f}=\text { specified }
\end{array}\right.\right.
$$

The inequality path constraints on control, state, and performance parameters of the aircraft are defined in Table 1.

\subsection{Simple VTM case studies}

Consider an aircraft flying toward a tall thin obstacle of height $h_{\text {obs }}$. This obstacle can be modeled as a bar of height $h_{\text {obs }}$. The mapping function is expressed as in

$$
f(x(\tau), u(\tau)) \triangleq \frac{t_{f}-t_{0}}{2}\left[\begin{array}{c}
\operatorname{Re}\left(C_{1}\left(\gamma\left(w-a_{1}\right)^{\alpha_{1}-1} \ldots\left(w-a_{n}\right)^{\alpha_{n}-1}\right)(V \cos \gamma+i V \sin \gamma)\right) \\
I M\left(C_{1}\left(\gamma\left(w-a_{1}\right)^{\alpha_{1}-1} \ldots\left(w-a_{n}\right)^{\alpha_{n}-1}\right)(V \cos \gamma+i V \sin \gamma)\right) \\
T_{\max }(M, y) g(\eta) \cos \alpha-D-m g \sin \gamma \\
T_{\max }(M, y) g(\eta) \sin \alpha+L-m g \cos \gamma
\end{array}\right]
$$


Table 1. State, control, and performance parameters for path constraints.

\begin{tabular}{cccl}
\hline Row & Symbol & Constraints description & Value \\
\hline 1 & $\alpha_{\min }$ & Minimum angle of attack & $-10^{\circ}$ \\
2 & $\alpha_{\max }$ & Maximum angle of attack & $+15^{\circ}$ \\
3 & $\gamma_{\min }$ & Minimum flight path angle & $-70^{\circ}$ \\
4 & $\gamma_{\max }$ & Maximum flight path angle & $+70^{\circ}$ \\
5 & $\dot{h}_{\min }$ & Maximum rate of descent & $-100 \mathrm{~m} / \mathrm{s}$ \\
6 & $\dot{h}_{\max }$ & Maximum rate of climb & $+120 \mathrm{~m} / \mathrm{s}$ \\
7 & $V_{\min }$ & Minimum aircraft speed & $70 \mathrm{~m} / \mathrm{s}$ \\
8 & $V_{\max }$ & Maximum aircraft speed & $300 \mathrm{~m} / \mathrm{s}$ \\
\hline
\end{tabular}
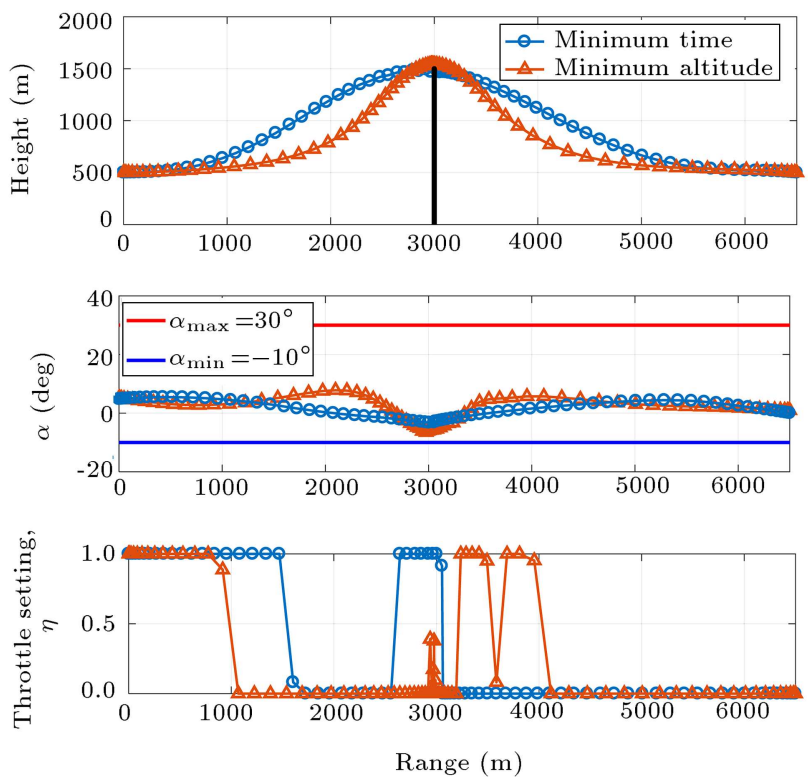

Figure 6. Aircraft optimal trajectories and control inputs (slim bar).

Eq. (16). Based on Eq. (18), taking the time derivative of Eq. (16), the transformed equation of motion in the mapped space could be obtained as follows:

$$
\dot{w}=\frac{\sqrt{w^{2}-1}}{s w}(V \cos \gamma+i V \sin \gamma) .
$$

The numerical solution of the obtained optimal TF/TA problem with the aforementioned mathematical models, constraints, and cost function will result in the optimal trajectory. The aircraft is assumed to be starting its maneuver from a trimmed level flight. The problem has been solved for all mission scenarios based on both minimum flight time (i.e., $K=1$ ) and minimum flying altitude (i.e., $K=0$ ).

The problem has been solved for a slim bar with a height of $1500 \mathrm{~m}$. Figure 6 illustrates the planned trajectory along with the corresponding control inputs with respect to aircraft downrange. The bang-bang throttle setting is based on the constrained optimal control problem, and generally shows that the speed will change during the flight maneuver. The time durations of flight for the minimum time and altitude scenarios are 41.1 and 49.8 seconds, respectively. Figure 7 shows the corresponding flight path angle and speed of the aircraft. Figure 8 shows the rate of changes in a vehicle's altitude that lies between the maximum RoC and RoD values.

In the case of a triangular VTM, the mapped equation of motion is expressed as follows based on Eqs. (13) and (19):

$$
\begin{gathered}
\dot{w}=\frac{1}{C_{1}}\left[\frac{\left(w-a_{1}\right)^{\alpha_{1}}\left(w-a_{3}\right)^{\alpha_{3}}}{(w)^{\alpha_{2}}}\right] \\
(V \cos \gamma+i V \sin \gamma) .
\end{gathered}
$$

The optimal trajectory for a triangular terrain model is shown in Figure 9. In the case of the minimum flying altitude scenario, the resulting path shows a tendency to keep itself in the neighborhood of the VTM. Figure 10 shows the corresponding flight speed and path angle, and the rate of changes in height is shown in Figure 11. The time durations of flights for this scenario are 38 and 49 seconds for time- and heightoptimal trajectories. The shape of the triangular VTM is the only cause of different trajectories in the same scenarios with invariant initial and final conditions. Therefore, different paths can be distinguished by three different parameters since each triangle is specified with three parameters.

\subsection{Complex terrain models}

As mentioned before, the Schwarz-Christoffel theorem could be utilized to model complex terrain profiles using polygonal shapes. However, the problem is that the numerical computation of these models increases by increasing the number of edges (sides) of the polygon. Here, we have used a code for computing some more 

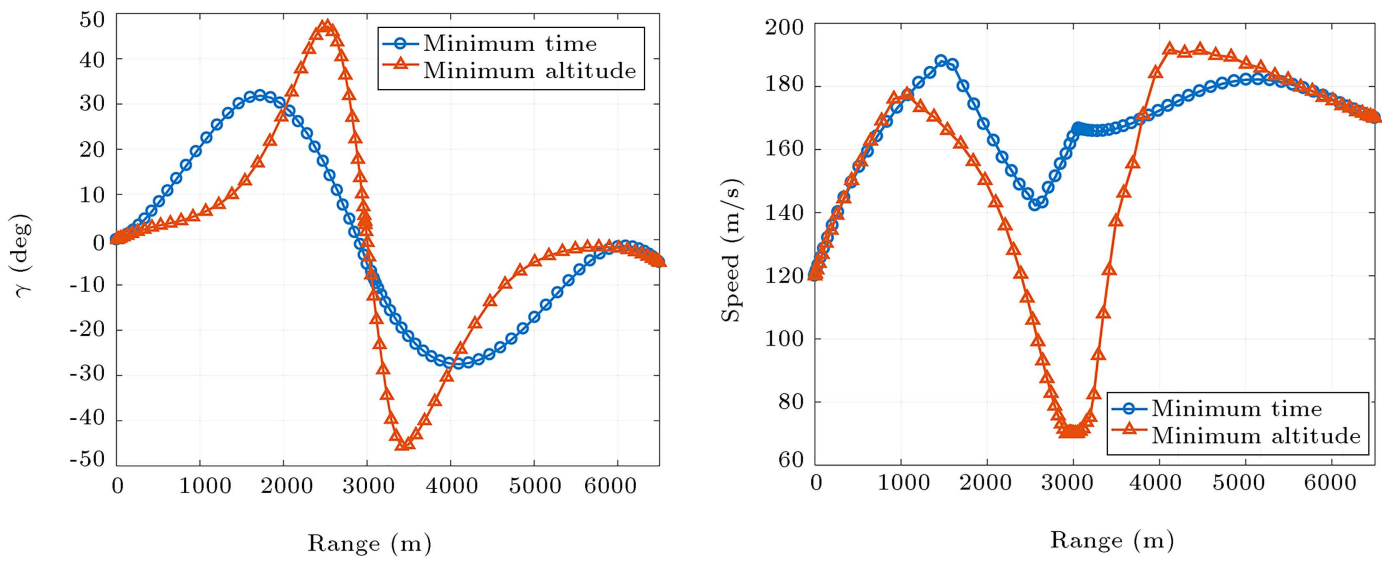

Figure 7. Aircraft flight path angle and speed (slim bar).

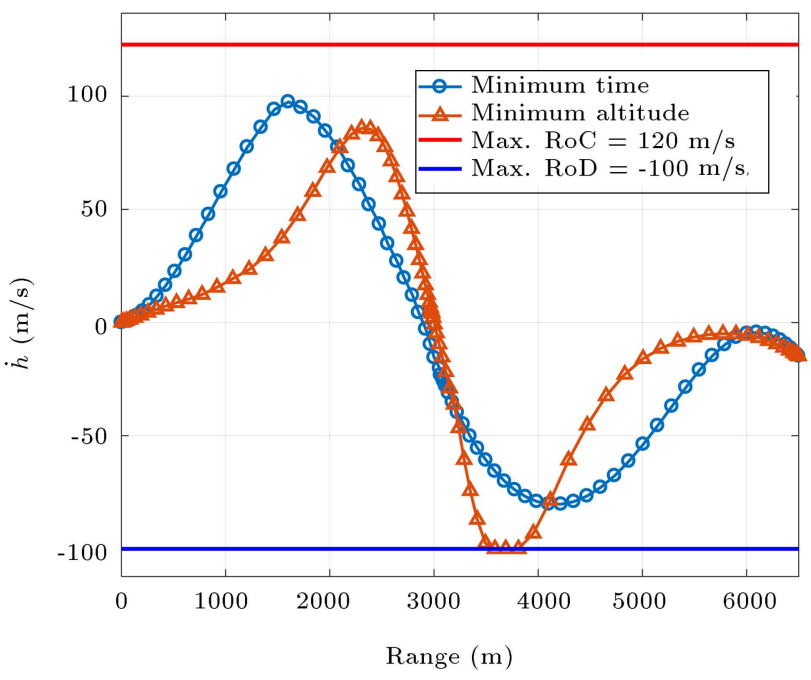

Figure 8. Aircraft rate of changes in height (slim bar).
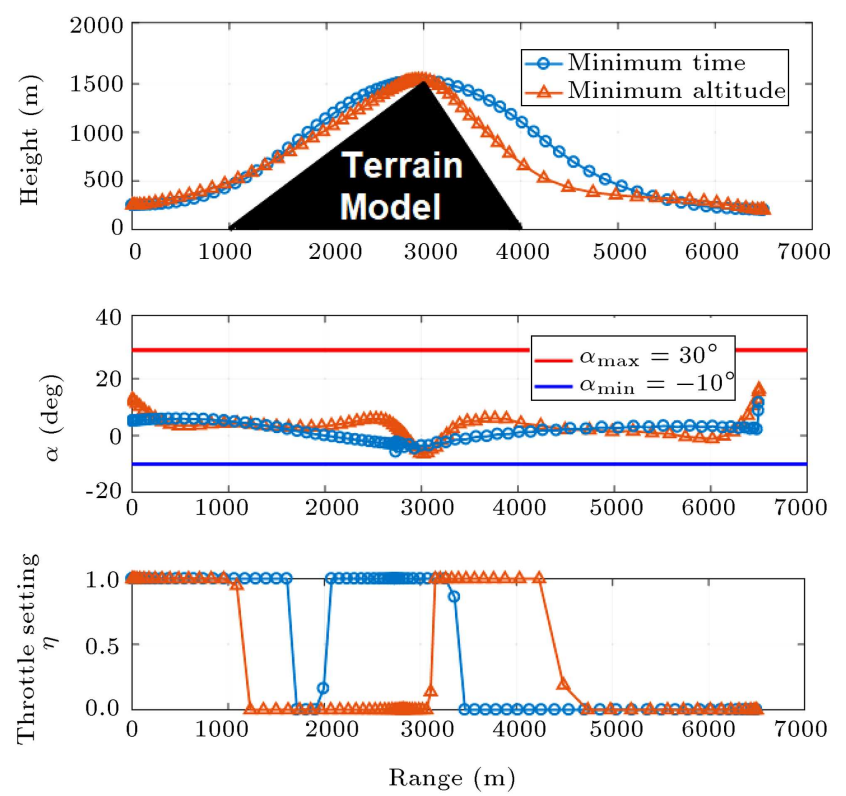

Figure 9. Aircraft optimal trajectories and control inputs (triangular Virtual Terrain Model (VTM)). complex mapping functions, which is under further development.

Suppose that the terrain profile has been estimated with a polygonal shape with five vertices. The mapped equation of motion is then expressed as follows:

$$
\begin{aligned}
\dot{w}= & C_{1}\left[\frac{\left(w-a_{2}\right)^{\alpha_{2}}\left(w+a_{2}\right)^{\alpha_{2}}}{\left(w-a_{1}\right)^{\alpha_{1}}(w)^{\alpha_{3}}\left(w+a_{1}\right)^{\alpha_{1}}}\right] \\
& (V \cos \gamma+i V \sin \gamma) .
\end{aligned}
$$

The parameters $\alpha_{1}, \alpha_{2}$, and $\alpha_{3}$ are shown in Figure 12 . One of the parameters of $a_{1}$ and $a_{2}$ is chosen arbitrarily, and the other one along with $C_{1}$ is calculated by the code. The VTM properties are presented as follows: $h_{1}=1250 \mathrm{~m}, h_{2}=100 \mathrm{~m}, k_{1}=300 \mathrm{~m}$, and $k_{2}=$ $1300 \mathrm{~m}$.

The resulting flight trajectories for time- and height-optimal cases are shown in Figure 13. Figure 14 illustrates the corresponding flight path angle and speed history with respect to the aircraft's downrange. The minimum flight time path exhibits a tendency to track a straight flight path to the destination. Therefore, the aircraft tries to increase its height and velocity using the maximum throttle and, after reaching the maximum altitude, the throttle setting is maximized for the second time to reach the final position in a minimum amount of time. In the minimum flying altitude case, the aircraft's throttle setting switches multiple times between maximum and minimum values to keep its path near the VTM profile. Figure 15 depicts the importance of considering the aircraft maximum RoC and RoD in the trajectoryplanning process. It shows how the aircraft's altitude change rate has been confined within the maximum and minimum possible values.

\subsection{Solution computation time}

One of the important requirements of a trajectory planner is the capability of generating fast optimal and feasible paths, such that the algorithm could be 

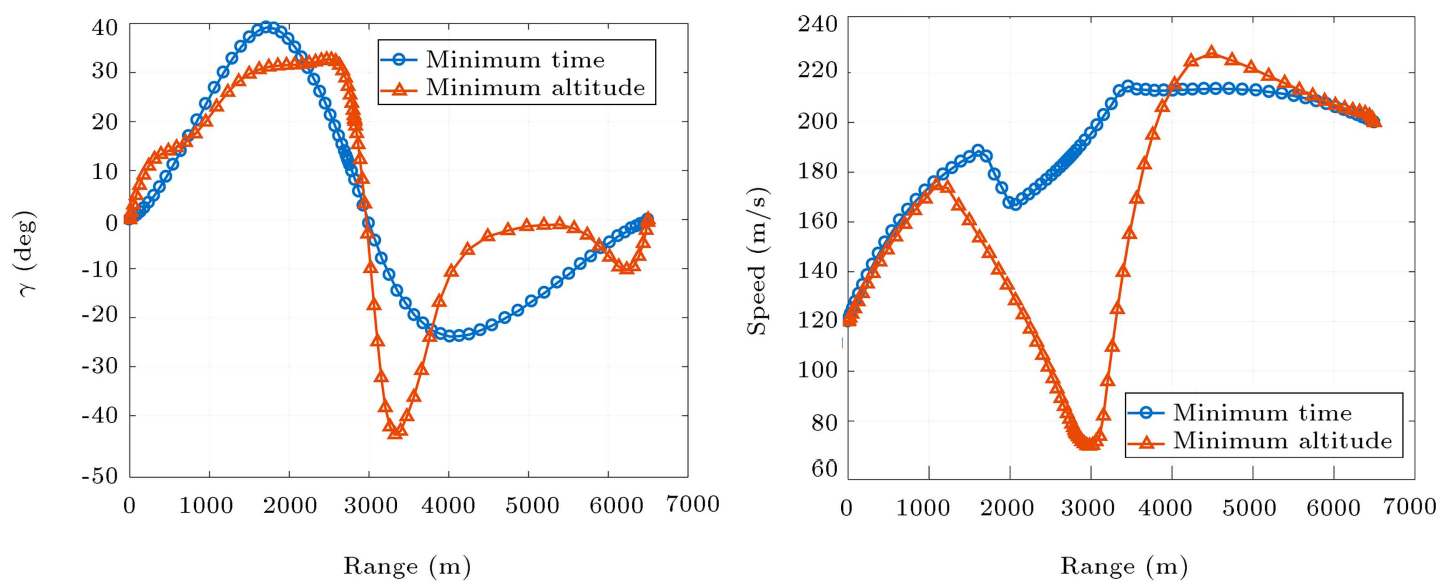

Figure 10. Aircraft flight path angle and speed (triangular Virtual Terrain Model (VTM)).

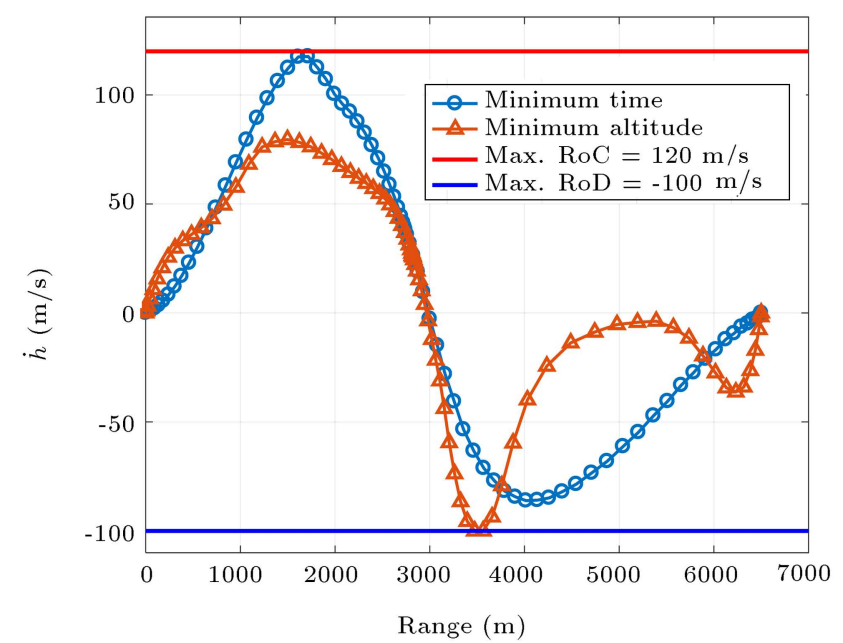

Figure 11. Aircraft rate of change of height (triangular Virtual Terrain Model (VTM)).

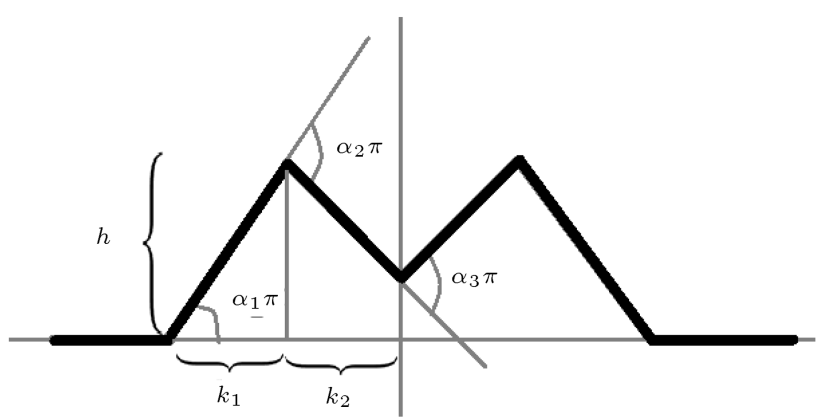

Figure 12. An example of a complex terrain model.

used in an online manner. Multiple case studies for different VTMs show that the solution computation time is around a few seconds when the initial guess of the NLP algorithm is not so accurate. Table 2 shows the mean solution computation time of TF/TA trajectory planning for a typical flight scenario of about 50-second duration for various VTM types, resulting from multiple runs. The optimization software runs
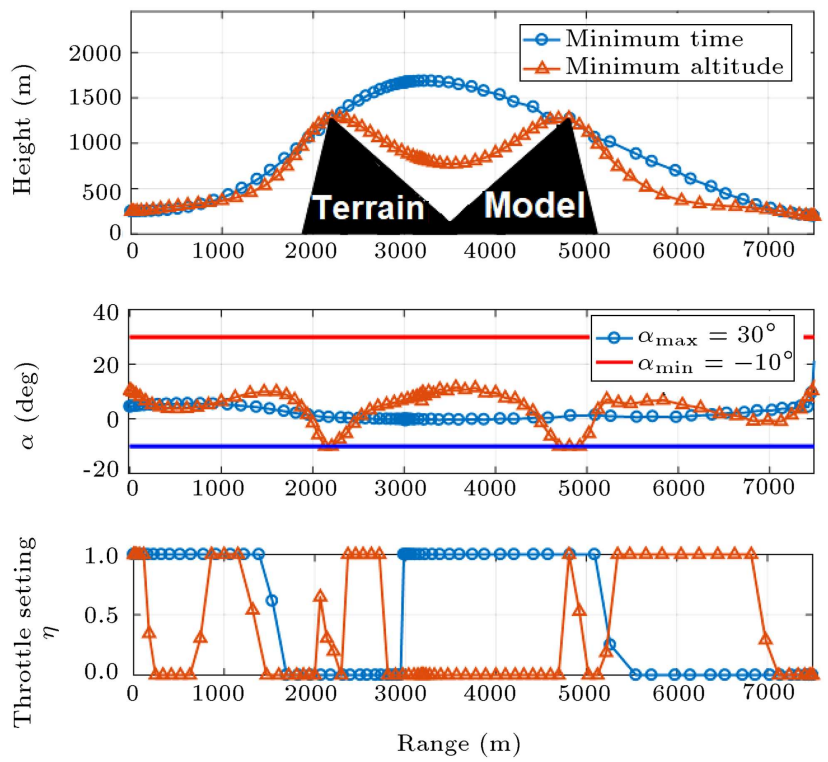

Figure 13. Aircraft optimal trajectories and control inputs (complex Virtual Terrain Model (VTM)).

Table 2. Solution computation time.

\begin{tabular}{ccc}
\hline Row & VTM type & $\begin{array}{c}\text { Mean computation } \\
\text { time }(\mathbf{s e c})\end{array}$ \\
\hline 1 & Straight bar & 2.0 \\
2 & Triangle & 4.5 \\
3 & $\begin{array}{c}\text { Two consecutive } \\
\text { triangles }\end{array}$ & 6.0 \\
\hline
\end{tabular}

were performed on regular legacy hardware and highlevel software MATLAB ${ }^{\complement}$.

The case studies show that the computational time can be significantly reduced if a more powerful initial guess can be exploited. In this regard, one solution to this problem is the use of limited storage solutions in the flight computer. 

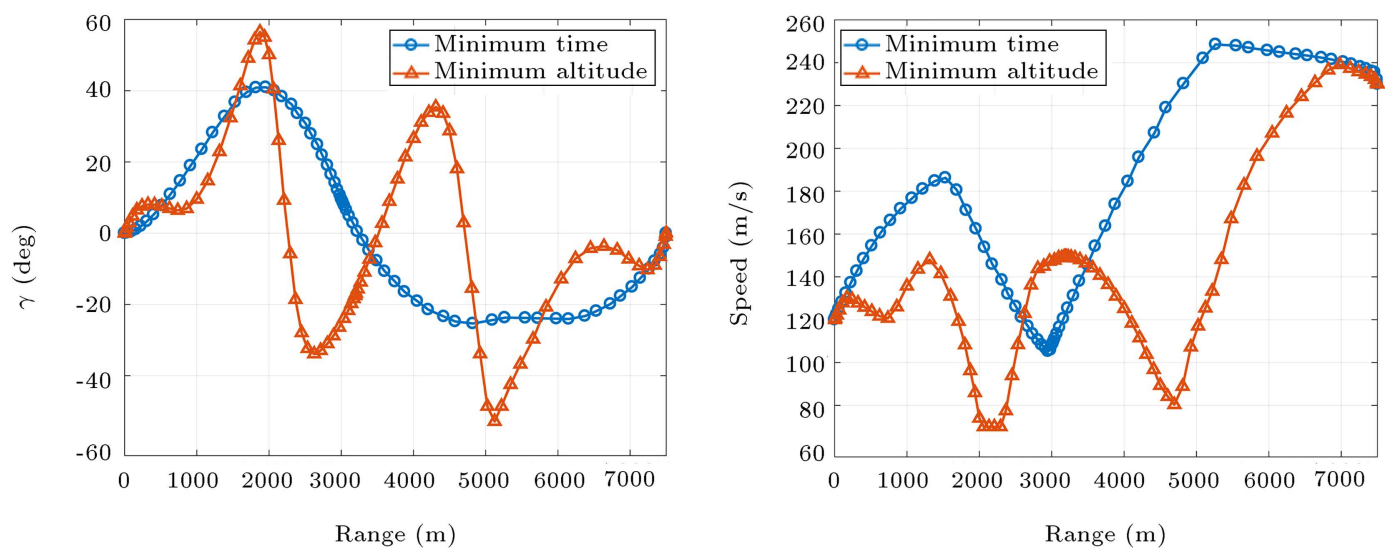

Figure 14. Aircraft flight path angle and speed (complex Virtual Terrain Model (VTM)).

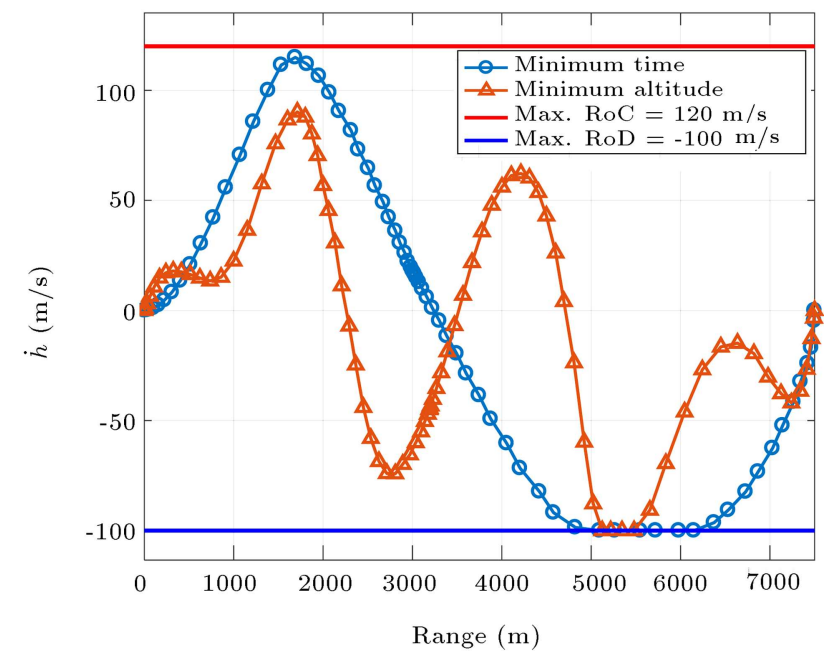

Figure 15. Aircraft rate of changes in height (complex Virtual Terrain Model (VTM)).

\section{Conclusion and future works}

The development of aircraft guidance and control system with Terrain Following (TF)/Terrain Avoidance (TA) capabilities is quite a difficult and timeconsuming process. One of the main issues pertaining to developing such a system is the trajectory-planning strategy and algorithm. The main problem in TF/TA trajectory planning is how the aircraft flight computer can generate a safe trajectory in accordance with the aircraft dynamic and flight performance capabilities. This research attempted to propose a solution approach to tackle this problem.

This paper presented an optimal 2D trajectoryplanning strategy for TF/TA flights. In the proposed strategy, by introducing the Schwarz-Christoffel theorem, the concept of conformal mapping, as a configuration space flattener, was utilized to transform the constrained vehicle's real motion configuration space into an obstacle-free mapped cyberspace. The regenerated transformed optimal control problem with multiple dynamic path constraints was solved using the direct LGR pseudo-spectral method.

Different case studies and simulation results showed that the proposed strategy was capable of generating fast and feasible solutions. By combining the obstacle detection sensors data with the terrain digital map data, the inputs required to implement the strategy were provided. Therefore, the proposed approach has the potential to be used in the aircraft Terrain Avoidance Warning System (TAWS), providing the pilot with quantitative and qualitative advice in the case of encountering an obstacle.

Since one of the challenges in the Unmanned Aerial Vehicle (UAV) path planning is the applicability of that strategy in the presence of partial knowledge of environment while maintaining feasibility, it is proposed that semi-analytical methods be applied in the solution process. In this regard, one of the issues that the authors are working on is the implementation of this strategy in an online manner and a closed-loop form. Moreover, computation time delays in beginning an obstacle-avoidance maneuver need to be taken into account in online applications when the path is required to be updated to avoid detected objects or terrain. Therefore, another future approach is to utilize this strategy for online applications.

\section{References}

1. International Civil Aviation Organization, Safety Report. A Coordinated, Risk-based Approach to Improving Global Aviation Safety, Montréal, Canada (2014).

2. Nonami, K., Kendoul, F., Suzuki, S., Wang, W., and Nakazawa, D. "Introduction", In Autonomous Flying Robots, pp. 1-29, Springer, Tokyo, Japan (2010).

3. Shiller, Z. "Off-line and on-line trajectory planning", In Motion and Operation Planning of Robotic Systems, G. Carbone and F. Gomez-Barvopp, Eds., pp. 29-62, Springer, New York (2015).

4. Kirk, D.E. "The variational approach to optimal 
control problems", In Optimal Control Theory: An Introduction, Dover Publication Inc., New York (2004).

5. Yang, H. and Zhao, Y. "Trajectory planning for autonomous aerospace vehicles amid known obstacles and conflicts", Journal of Guidance, Control, and Dynamics, 27(6), pp. 997-1008 (2004).

6. Vachtsevanos, G., Tang, L., Drozeski, G., and Gutierrez, L. "From mission planning to flight control of unmanned aerial vehicles: Strategies and implementation tools", Annual Reviews in Control, 29(1), pp. 101-115 (2005).

7. Karelahti, J., Virtanen, K., and Öström, J. "Automated generation of realistic near-optimal aircraft trajectories", Journal of Guidance, Control, and Dynamics, 31(3), pp. 674-688 (2008).

8. Lee, S., Cho, A., and Kee, C. "Integrated waypoint path generation and following of an unmanned aerial vehicle", Aircraft Engineering and Aerospace Technology, 82(5), pp. 296-304 (2010).

9. Babaei, A.R. and Mortazavi, M. "Fast trajectory planning based on in-flight waypoints for unmanned aerial vehicles", Aircraft Engineering and Aerospace Technology, 82(2), pp. 107-115 (2010).

10. Lin, Z., Castano, L., and Xu, H. "A fast obstacle collision avoidance algorithm for fixed wing UAS", 2018 International Conference on Unmanned Aircraft Systems (ICUAS), Tokyo, Japan, pp. 559-568 (2018).

11. Naidu, D.S. "Constrained optimal control systems", In Optimal Control Systems, CRC Press LLC, Washington, D.C, pp. 293-361 (2003).

12. Menon, P.K.A., Cheng, V.H.L., and Kim, E. "Optimal trajectory synthesis for terrain-following flight", Journal of Guidance, Control, and Dynamics, 14(4), pp. 807-813 (1991).

13. Malaek, S.M. and Kosari, A. "Dynamic based cost functions for TF/TA flights", IEEE Transactions on Aerospace and Electronic Systems, 48(1), pp. 44-63 (2012).

14. Malaek, S.M. and Kosari, A. "Novel minimum time trajectory planning in terrain following flights", IEEE Transactions on Aerospace and Electronic Systems, 43(1), pp. 2-12 (2007).

15. Kosari, A. and Kassaei, S.I. "Dynamic constrained low altitude flight maneuvers with constant energy", 2017 IEEE International Conference on Power, Control, Signals and Instrumentation Engineering (ICPCSI), India, pp. 3085-3089 (2017).

16. Denton, J.E. and Jones, R.V. "A new technique for terrain following/terrain avoidance guidance command generation", 40th AGARD Guidance and Control Symposium, New York (1985).

17. Jalali-Naini, S.H. and Sajjadi, S.H. "First-order optimal line-of-sight guidance for stationary Targets", Scientia Iranica, 23(2), pp. 588-599 (2016).
18. Kosari, A. Maghsoudi, H., and Lavaei, A. "Path generation for flying robots in mountainous regions", International Journal of Micro Air Vehicles, 9(1), pp. 44-60 (2017).

19. Williams, P. "Three-dimensional aircraft terrainfollowing via real-time optimal control", Journal of Guidance, Control, and Dynamics, 30(4), pp. 12011206 (2007).

20. Kamyar, R. and Taheri, E. "Aircraft optimal terrain/threat-based trajectory planning and control", Journal of Guidance, Control, and Dynamics, 37(2), pp. 466-483 (2014).

21. Qing, L. "Aircraft route optimization using genetic algorithms", Second International Conference on Genetic Algorithms in Engineering Systems, Glascow, UK, pp. 394-397 (1997).

22. Nikolos, I.K., Valavanis, K.P., Tsourveloudis, N.C., and Kostaras, A.N. "Evolutionary algorithm based offline/online path planner for uav navigation", IEEE Transactions on Systems, Man and Cybernetics, Part $B$ (Cybernetics), 33(6), pp. 898-912 (2003).

23. Nikolos, I.K. and Tsourvelouds, N.C. "Path planning for cooperating unmanned vehicles over 3-D terrain", Informatics in Control, Automation and Robotics: Selected Papers from the International Conference on Informatics in Control, Automation and Robotics 2007, Berlin, pp. 153-168 (2009).

24. Sun, T.Y., Huo, C.L., Tsai, S.J., and Liu, C.C. "Optimal UAV flight path planning using skeletonization and particle swarm optimizer", 2008 IEEE Congress on Evolutionary Computation (IEEE World Congress on Computational Intelligence), Hong Kong, Chin, pp. 1183-1188 (2008).

25. Karimi, J. and Pourtakdoust, S.H. "Optimal maneuver-based motion planning over terrain and threats using a dynamic hybrid PSO algorithm", Aerospace Science and Technology, 26(1), pp. 60-71 (2013).

26. Nobahari, H. and Arab Kermani, M. "Integrated optimization of guidance and control parameters in a dual spin flying vehicle", Scientia Iranica, 24(5), pp. 2473-2489 (2017).

27. Kosari, A., Maghsoudi, H., Lavaei, A., and Ahmadi, R. "Optimal online trajectory generation for a flying robot for terrain following purposes using neural network", Proceedings of the Institution of Mechanical Engineers, Part G: Journal of Aerospace Engineering, 229(6), pp. 1124-1141 (2015).

28. Rahim, M. and Malaek, S.M. "Intelligent operation using terrain following flight in unmanned aerial vehicles", 2007 IEEE Aerospace Conference, Big Sky, MT, USA, pp. 1-8 (2007).

29. Rahim, M. and Malaek, S.M. "Aircraft terrain following flights based on fuzzy logic", Aircraft Engineering and Aerospace Technology, 83(2), pp. 94-104 (2011). 
30. Bagherian, M. "Unmanned aerial vehicle terrain following/terrain avoidance/threat avoidance trajectory planning using fuzzy logic", Journal of Intelligent \& Fuzzy Systems, 34(3), pp. 1791-1799 (2018).

31. Vachhani, L., Kumar, P.R., and Seridharan, "Advances in mapless and map-based strategies for robot navigation a review", In Robot Vision: Strategies, Algorithms and Motion Planning, K.D. Ito, Ed., Nova Science Publishers, Inc., New York (2009).

32. Megherbi, D. and Wolovich, W.A. "Real-time velocity feedback obstacle avoidance via complex variables and conformal mapping", Proceedings 1992 IEEE International Conference on Robotics and Automation, Nice, France, pp. 206-213 (1992).

33. Chuang, J.H. and Ahuja, N. "An analytically tractable potential field model of free space and its application in obstacle avoidance", IEEE Transactions on Systems, Man and Cybernetics, Part B (Cybernetics), 28(5), pp. 729-736 (1998).

34. Chen, Y., Luo, G., Mei, Y., Yu, J., and Su, X. "UAV path planning using artificial potential field method updated by optimal control theory", International Journal of Systems Science, 47(6), pp. 1407-1420 (2016).

35. Qu, Y., Zhang, Y., and Zhang, Y. "A global path planning algorithm for fixed-wing UAVs", Journal of Intelligent \& Robotic Systems, 91(3), pp. 691-707 (2018).

36. Kosari, A. and Kassaei, S.I. "Using conformal mapping in developing a novel optimal obstacle-avoidance trajectory-planning for a flying robot", 2017 IEEE International Conference on Power, Control, Signals and Instrumentation Engineering (ICPCSI), India, pp. 3080-3084 (2017).

37. Roskam, J. "General equations of motion for rigid airplanes", In Airplane Flight Dynamics and Automatic Flight Controls, pp. 9-46, Roskam Aviation and Engineering Corporation, Lawrence (2001).

38. Brown, J.W. and Churchill R.V. "Conformal mapping", In Complex Variables and Applications, 8th Edn., pp. 355-372, McGraw-Hill, Michigan (2008).

39. Abolwitz, M.J. and Fokas, A.S. "Conformal mappings and applications", In Complex Variables Introduction and Applications, 2nd Edn., pp. 311-408, Cambridge University Press, New York (2003).

40. Driscoll, T.A. and Trefethen, L.N. "Numerical methods", In Schwarz-Christoffel Mapping, pp. 23-39, Cambridge University Press, New York (2003).

41. Faires, J.D. and Burden, R. "Numerical solutions of nonlinear systems of equations", in Numerical Analysis, 9th Edn., pp. 629-669, Brooks/Cole Cengage Learning, New York (2010).
42. Rao, A.V. "Survey of numerical methods for optimal control", Advances in the Astronautical Sciences, 135(1), pp. 497-528 (2009).

43. Betts, J.T. "Survey of numerical methods for trajectory optimization", Journal of Guidance, Control, and Dynamics, 21(2), pp. 193-207 (1998).

44. Huntington, G.T. "Advancement and analysis of a gauss pseudospectral transcription for optimal control problems", PhD Thesis, Department of Aeronautics and Astronautics, Massachusetts Institute of Technology (2007).

45. Garg, D., Patterson, M., Hager, W., Rao, A.V., Benson, D.A., and Huntington, G.T. "A unified framework for the numerical solution of optimal control problems using pseudospectral methods", Automatica, 46(11), pp. 1843-1851 (2010).

46. Garg, D., Hager, W., and Rao, A.V. "Pseudospectral methods for solving infinite-horizon optimal control problems", Automatica, 47(4), pp. 829-837 (2011).

47. Gill, P.E., Murray, W., and Saunders, M.A. "SNOPT: an SQP algorithm for large-scale constrained optimization", SIAM Journal on Optimization, 12(4), pp. 9791006 (2002).

48. Khademi, I., Maleki, B., and Nasseri Mood, A. "Optimal three dimensional terrain following/terrain avoidance for aircraft using direct transcription method", 2011 19th Mediterranean Conference on Control \& Automation (MED), Corfu, Greece, pp. 254-258 (2011).

\section{Biographies}

Amirreza Kosari is an Associate Professor of University of Tehran in Iran. His research interests include trajectory optimization, optimal control, cooperative flights, and spacecraft attitude control. He is the author of several papers in the areas indicated above. Dr. Kosari obtained his BS degree in 1998 from Amirkabir University, Tehran, Iran, his MS degree in 2001, and his $\mathrm{PhD}$ in 2008 from Sharif University of Technology, Tehran, Iran.

Seyed Iman Kassaei received his BS degree in Aerospace Engineering from Sharif University of Technology, Tehran, Iran in 2007 and his MS degree from Khaje Nasir Toosi University, Tehran, Iran in 2011. Currently, he is now PhD student in Aerospace Engineering at the Faculty of New Sciences and Technologies, University of Tehran, Tehran, Iran. His research interests include aircraft design and performance analysis, unmanned vehicles guidance, and navigation. 